\title{
Averaging rheological quantities in descriptions of soft glassy materials
}

\author{
François Lequeux ${ }^{1}$, Armand Ajdari ${ }^{2}$ \\ ${ }^{1}$ Laboratoire de Physico-Chimie Macromoléculaire, UMR CNRS 7615 \\ ${ }^{2}$ Laboratoire de Physico-Chimie Théorique, Esa CNRS 7083 \\ both at : ESPCI, 10 rue Vauquelin, F-75231 Paris Cedex 05, France \\ submitted to Phys.Rev.E, Nov. 27, 2000
}

\begin{abstract}
Many mean-field models have been introduced to describe the mechanical behavior of glassy materials. They often rely on averages performed over distributions of elements or states. We here underline that averaging is a more intricate procedure in mechanics than in more classical situations such as phase transitions in magnetic systems. This leads us to modify the predictions of the recently proposed SGR model for soft glassy materials, for which we suggest that the viscosity should diverge at the glass transition temperature $T_{g}$ with an exponential form $\eta \sim \exp \left(\frac{A}{T-T_{g}}\right)$.

PACS: 62.20.Fe, 64.70.Pf, 83.80.Hj
\end{abstract}

\section{INTRODUCTION}

Concentrated colloidal suspensions of soft particles (emulsions, slurries) tend to display glassy behavior when concentrated beyond a threshold [1 5. Despite their great physical and chemical diversity, these Soft Glassy Materials often share many mechanical features. This has prompted theoreticians to construct generic models [6 10], independent of the microscopic features of the systems, and often borrowed from statistical physics. The outcome of such a procedure is often a description in terms of a large distribution of relaxation processes acting simultaneously, a picture supported by experimental facts for many glassy systems [11,12].

Any macroscopic mechanical response of the system then results from an averaging over these distributions. In this paper we stress the importance of the averaging procedure when one deals with mechanics. Averaging procedures have already been discussed for nonmechanical situations in the non-ergodic glassy phase (e.g. statistical mechanics of spin glasses [11]). However averaging of mechanical fields is a priori a different story [13]. This also affects the description of the ergodic fluid phase (e.g. the divergence of the viscosity when one approaches the "glass transition" from the fluid side).

To make our point we focus here on the recently introduced SGR model (Soft Glassy Rheology) [6] which incorporates mechanics in a simple picture of the glass transition introduced by Bouchaud. The system is taken as a collection of blocks, that evolve according to stochastic equations, driven by the imposed shear rate. A control parameter of the model is the effective temperature that we will denote here $T$. A transition occurs at $T_{g}$ below which the system is no more ergodic (and the fluid displays a yield stress). A remarkable feature of the model is that the viscosity diverges at $2 T_{g}$ (not $T_{g}$ ), so that in the interval $T_{g}<T<2 T_{g}$ the material is a power-law fluid. This large domain of viscosity divergence, in the ergodic phase, is a feature absent in most other models for soft glassy materials [0], 8,10$]$.

After general comments about averaging procedures in mechanics (section II), we show below that this feature is a consequence of the adopted averaging procedure (section III). We propose an improvement of this procedure, that leads to a Vogel-Fulcher divergence of the viscosity at $T_{g}: \eta \sim \exp \left(\frac{A}{T-T_{g}}\right)$. We end by a short discussion and by drawing a few research directions (section IV).

\section{VARIOUS AVERAGES FOR RHEOLOGY/MECHANICS}

Let us start by clarifying the crucial role of the averaging procedure in rheology or mechanics, very different from the one used in many classical areas, such as magnetic systems, where the imposed field $H$ is well-defined , and the average magnetization is simply the algebraic mean of the local magnetizations. In rheological problems, the fields are imposed through the boundaries of the system, so that often the shear rate and the shear stress play more or less symmetric roles, which results in a wide variety of situations.

To clarify the message, let us focus on the simple case of a system built of elements (blocks) of high viscosity $\eta_{1}$ and elements (blocks) of low viscosity $\eta_{2}$ (Figs. 1a and $1 \mathrm{~b}$ ), and estimate the average viscosity of the system through different averaging procedures.

A first average corresponds to the situation where the same shear rate $\dot{\gamma}$ is imposed to all elements, and one averages the resulting stresses, which leads to an average viscosity

$$
\langle\eta\rangle_{\dot{\gamma}}=\phi_{1} \eta_{1}+\left(1-\phi_{1}\right) \eta_{2}
$$

where $\phi_{1}$ is the proportion of blocks of high viscosity. Strictly speaking this procedure describes blocks arranged in layers disposed in parallel (Fig. 1c). A second kind of average corresponds to submitting all blocks to 


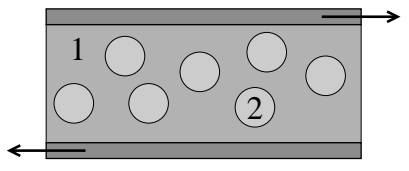

a

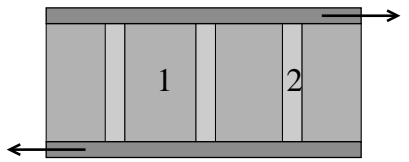

c

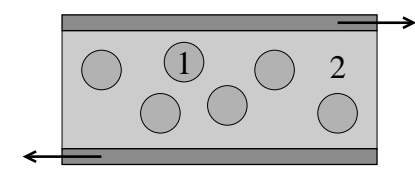

b

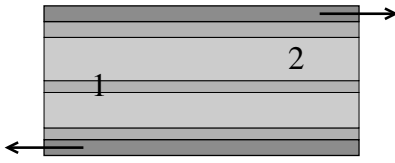

d
FIG. 1. Schematic pictures of : (a) a dispersion of fluid inclusions 2 in a highly viscous matrix 1, (b) a dispersion of highly viscous inclusions 1 in a fluid matrix 2, (c) a geometry corresponding to averaging at fixed strain rate $\dot{\gamma}$, which leads to a highly viscous behavior as in (a), (d) a geometry corresponding to averaging at fixed stress with a qualitative behavior similar to (b).

the same stress $\sigma$, and averaging the shear rates. This results in a different average viscosity

$$
\langle\eta\rangle_{\sigma}=\left(\phi_{1} \eta_{1}^{-1}+\left(1-\phi_{1}\right) \eta_{2}^{-1}\right)^{-1}
$$

which describes layers in series (Fig. 1d).

The behavior according to the first kind of average is dominated by the highly viscous blocks: the average viscosity $\langle\eta\rangle_{\dot{\gamma}}$ diverges if $\eta_{1}$ diverges, whatever the relative volume fractions. This is obviously a reasonable representation of a highly viscous medium with low-viscosity inclusions ( $\phi_{1}$ large, Fig. 1a), but a poor one for a system of highly viscous inclusions dispersed in a fluid matrix of low viscosity $\eta_{2}$ (Fig. 1b). On the contrary, the latter case $\left(\phi_{1}\right.$ small, Fig. 1b) is better represented by a fixed stress average (Fig. 1d), as $\langle\eta\rangle_{\sigma}$ is dominated by the low viscosity blocks.

Clearly none of these two simple averages properly describes all situations. Consequently effective medium theories have tried to provide better alternatives. Among those, let us quote the Olroyd-Palierne formula [14] for a matrix of viscosity $\eta_{m}$ with various kind of dilute spherical inclusions $i$ (volume fraction $\phi_{i}$, viscosity $\eta_{i}$ ). This formula states that the effective viscosity of the resulting composite fluid is (neglecting surface tension effects):

$$
\langle\eta\rangle_{O P}=\eta_{m} \frac{1+\frac{3}{2} \Sigma_{i} \phi_{i} H_{i}}{1-\Sigma_{i} \phi_{i} H_{i}}
$$

with

$$
H_{i}=\frac{2 \eta_{i}-2 \eta_{m}}{2 \eta_{i}+3 \eta_{m}}
$$

The above formula provides a convenient interpolation between the two limiting physics mentioned above. For a sparse dispersion of highly viscous blocks in a less viscous matrix (Fig1.b), it yields the Einstein formula $\langle\eta\rangle_{O P} \simeq \eta_{2}\left(1+\frac{5}{2} \phi_{1}\right)$, and thus similar physics to the constant stress picture $\langle\eta\rangle_{\sigma} \simeq \eta_{2}\left(1+\phi_{1}\right)$ (Fig. 1d). For a sparse dispersion of fluid inclusions in a highly viscous matrix (Fig. 1a), $\langle\eta\rangle_{O P} \simeq \eta_{1}\left(1-\frac{5}{3} \phi_{2}\right)$, a result qualitatively akin to that of the constant shear rate picture $\langle\eta\rangle_{\dot{\gamma}}=\eta_{1}\left(1-\phi_{2}\right)$ (Fig. 1c). Of course the Olroyd-Palierne formula is unable to describe accurately the properties of intermediate mixtures (roughly $\phi$ in the range $30-70 \%$ ) as it neglects spatial structures that could result in percolation effects of great consequence on the mechanical behavior.

\section{CONSEQUENCES FOR THE SGR MODEL}

Implicitly the SGR model of Sollich et al. [6] considers an ensemble of elements of viscosity distributed along:

$$
\eta(E)=\eta_{0} \exp (E / T)
$$

where $E$ characterizes the activation barrier limiting the yield of these blocks. The distribution of these barriers is modeled by $n(E) \sim \exp \left(-E / T_{g}\right)$, which naturally introduces $T_{g}$ as a (Glass) transition temperature. Indeed, for $T>T_{g}$, the states are populated according to Boltzmann statistics at steady state $p(E) \sim n(E) \exp (E / T)$ which gives $p(E)=\frac{T-T_{g}}{T_{g} T} \exp \left(-\frac{T-T_{g}}{T_{g} T} E\right)$, which ceases to be normalizable as $T \rightarrow T_{g}^{+}$.

The average viscosity used in this model is the constant rate one, which leads for $T>T_{g}$ to :

$$
\langle\eta\rangle_{\dot{\gamma}}=\int d E p(E) \eta(E)=\int_{0}^{\infty} d E \frac{T-T_{g}}{T T_{g}} e^{-\frac{T-2 T_{g}}{T T_{g}} E}
$$

which diverges for $T \rightarrow 2 T_{g}^{+}$(i.e. not for $T \rightarrow T_{g}^{+}$). With this averaging procedure, the viscosity is thus infinite in the range $T_{g}<T<2 T_{g}$, above the glass transition temperature. The corresponding mechanical behavior was found to be that of a power-law fluid with a temperature dependent exponent $\langle\sigma\rangle \sim \dot{\gamma}^{\frac{T-T_{g}}{T_{g}}}$.

Let us now explain why, on physical grounds, we expect this prediction to be incorrect, and the viscosity of the system to remain finite for $T_{g}<T<2 T_{g}$. For example, focus on the $10 \%$ most viscous blocks (i.e. those of viscosity larger than $\eta_{c}=\eta_{0} \exp \left(E_{c} / T\right)$ with $\left.E_{c}=-\frac{T_{g} T}{T-T_{g}} \log (0.1)\right)$. These few very viscous blocks are embedded in a matrix of viscosity smaller than $\eta_{c}$, as all its blocks have a viscosity weaker than $\eta_{c}$. The effective viscosity of the whole fluid, composed of this matrix with sparse quasi-solid inclusions, is thus smaller than $\eta_{c}$ times a geometric factor related to the $10 \%$ volume fraction (e.g. $\eta<\frac{1.15}{0.9} \eta_{c}$ in the Olroyd-Palierne model), and the actual position of the inclusions. The viscosity 
is thus finite for $T_{g}<T<2 T_{g}$ and can not diverge faster than $\eta_{c}=\eta_{0} \exp \left(\frac{T_{g}}{T-T_{g}} \log (10)\right)$ as $T \rightarrow T_{g}^{+}$.

Switching to the other limit and using a fixed stress average, leads to averaging the inverse of the viscosities (or the inverse of the relaxation times). The continuous version of Equation (2) yields an average viscosity:

$$
\langle\eta\rangle_{\sigma}=\eta_{0} \frac{T}{T-T_{g}}
$$

which displays a weak (algebraic) divergence at $T_{g}$

We now propose a physically sounder recipe based on the Olroyd-Palierne formula and a self-consistent approximation. We take a representative but small proportion $\phi$ of the blocks as "inclusions", while the rest is the "matrix". As the matrix and the whole system are similar, formula (3) gives an average viscosity $\langle\eta\rangle_{O P S S}=\eta_{m}$ :

$$
\langle\eta\rangle_{O P S S}=\langle\eta\rangle_{O P S S} \frac{1+\frac{3}{2} \Sigma_{i} \phi_{i} H_{i}}{1-\Sigma_{i} \phi_{i} H_{i}}
$$

with

$$
\Sigma_{i} \phi_{i} H_{i}=\phi \int_{0}^{\infty} d E p(E) \frac{2 \eta(E)-2\langle\eta\rangle_{O P S S}}{2 \eta(E)+3\langle\eta\rangle_{O P S S}}
$$

The average viscosity $\langle\eta\rangle_{O P S S}$ is thus implicitly given by $\Sigma_{i} \phi_{i} H_{i}=0$ (a similar approximation was used in another context 15]):

$$
\int_{0}^{\infty} d E p(E) \frac{5\langle\eta\rangle_{O P S S}}{2 \eta(E)+3\langle\eta\rangle_{O P S S}}=1
$$

Although the actual numerical factors ( 2 and 3 ) may be discussed, we believe that this formula gives a physically correct average, intermediate between the two limiting cases mentioned above: $\langle\eta\rangle_{\dot{\gamma}} \geq\langle\eta\rangle_{\text {OPSS }} \geq\langle\eta\rangle_{\sigma}$, and with the appropriate behavior in the two limits.

Applying this to the SGR model of Sollich et al. leads to a divergence of the viscosity at $T_{g}$. Indeed if we write the average viscosity at temperature $T$ in the form $\langle\eta\rangle_{\text {OPSS }}=\frac{2}{3} \eta_{0} \exp \left(E^{*} / T\right)$, then the implicit equation for $E^{*}$ is:

$$
\int_{0}^{\infty} d E p(E) \frac{1}{\exp \frac{E-E^{*}}{T}+1}=3 / 5
$$

Given that $\int_{0}^{\infty} d E p(E)=1$, this suggests $\int_{E^{*}}^{\infty} d E p(E) \sim$ $2 / 5$ and thus $\exp \left(-E^{*} \frac{T-T_{g}}{T_{g} T}\right) \sim 2 / 5$. This corresponds to a viscosity diverging for $T \rightarrow T_{g}^{+}$as $\sim$ $\frac{2}{3} \eta_{0} \exp \left(\frac{T_{g}}{T-T_{g}} \log (5 / 2)\right)$. This divergence, obtained by the above rough analysis, is confirmed by numerical inspection of (10) which gives

$$
\langle\eta\rangle_{O P S S} \sim \eta_{0} \exp \left(\frac{T_{g}}{T-T_{g}} \log (5 / 2)\right)
$$

with a prefactor $\simeq 0.72$ instead of $2 / 3$. This exponential divergence is consistent with our qualitative analysis below equation (6).

Of course the $5 / 2$ is not to be taken too seriously, but we think that a Vogel-Fulcher like divergence of the viscosity at $T_{g}$ is a sound result, within the hypothesis of the SGR model.

\section{DISCUSSION}

In conclusion, great care should be taken when choosing an averaging procedure for the mechanical properties of a system close to a "glass transition". Fixed stress and fixed shear rate averages are only extremes that limit a large set of possibilities. A physically appealing intermediate procedure for elements of distributed properties, adapted from effective medium theory, has been proposed in equation (10).

Focusing on the SGR model, we have shown that using different averages seriously modifies the law characterizing the divergence of the viscosity. Using the recipe of equation (10) we have proposed that a Vogel-Fulcher divergence could quite naturally arise. Revisiting the model, to incorporate the effects of the inhomogeneity of the driving strain rate field $\dot{\gamma}$ on the dynamics, is a direction that we wish to explore.

It would also be interesting to analyze thoroughly what kind of averages are implicitly made within the dynamical rules used in other models for such systems [7,8,10]. For example the simple models of [7,8] predict an algebraic divergence of the viscosity. A natural question is whether this is the result of an implicit averaging of the inverse of the relaxation times as in equation (7).

We have considered here the simplest (in principle) situation of an ergodic system (i.e. above the effective glass transition temperature), where neglecting spatial structures and adopting mean-field approaches is most likely to apply. Analysis of non-ergodic situations is left for further work. Eventually let us clarify that we have focused here on the sole viscosity to make our point clear, but that a more complete description of rheological properties would have to deal with effective theories for assemblies of visco-elastic (and possibly plastic) elements [14]. Such effective theories most likely will have to deal with the importance of spatial correlations and with the role of percolating structures.

Acknowledgments: we thank S. Obukhov for fruitful discussions on related issues. 
[1] T.G. Mason, J. Bibette, D.A. Weitz, Phys. Rev. Lett., 75, 2051-2054 (1995).

[2] T.G. Mason, J. Bibette, D.A. Weitz, J. Colloid. Interf. Sci., 179, 439-448 (1996).

[3] R.J. Ketz, R.K. Prudhomme, W.W. Graessley, Rheol. Acta, 27, 531-539 (1988).

[4] M. Cloitre, R. Borrega, L. Leibler, Phys.Rev.Let. to appear.

[5] C. Derec, A. Ajdari, G. Ducouret, F. Lequeux, C.R.Acad.Sci. Paris, to appear.

[6] P. Sollich, F.Lequeux, P. Hébraud, M.E. Cates, Phys. Rev. Lett., 78, 2020-2023 (1997). P. Sollich, Phys. Rev. E, 58, 738-759 (1998).

[7] P. Hébraud, F. Lequeux, Phys. Rev. Lett., 81, 2934-2937 (1998).

[8] C. Derec, A. Ajdari, F. Lequeux, Faraday Discussions, 112, 195-207 (1999).

[9] J. Kurchan, "Rheology, and how to stop aging", preprint CondMat 9812347.

[10] L. Berthier, J.L. Barrat, J. Kurchan, Phys. Rev. E, 61, 5464-5472 (2000).

[11] J.P. Bouchaud, L.F. Cugliandolo, M. Mézard, J. Kurchan, in Spin Glasses and Random Fields, ed. A.P. Young, World Scientific, Singapore 1997; and references therein.

[12] W. Götze, L. Sjögren, Rep. Prog. Phys., 55, 241 (1992).

[13] L. Golubovic, T.C. Lubensky,Phys. Rev. Lett., 63, 1082 (1989).

[14] J.F. Palierne, Rheol. Acta, 29204 (1990), and references therein.

[15] Y. Hemar, R. Hocquart, F. Lequeux, J. Phys. Fr., 5, 1576 (1995). 